\title{
Metastatic Colonic Adenocarcinoma Simulating Primary Ovarian Neoplasm in Transvaginal Doppler Sonography
}

\author{
Joel S. Newman, MD, and Ronald S. Adler, PhD, MD
}

Transvaginal color flow and pulsed Doppler sonography has shown promise in the evaluation and characterization of adnexal masses and may ultimately play a role in ovarian cancer screening. ${ }^{1,2}$ A relative increase in the diastolic flow in malignant lesions has been previously reported. Pathologic evidence suggests that this phenomenon reflects the paucity of smooth muscle within tumor vessels along with intratumoral arteriovenous shunting. ${ }^{3,4}$ We present a case of a postmenopausal woman who presented with large adnexal masses simulating primary ovarian neoplasm in gray-scale sonography that, in addition, manifested an abnormally low resistive index in the transvaginal Doppler examination. Further evaluation with computed tomography (CT) and, ultimately, laparotomy revealed metastatic spread of a mucinous adenocarcinoma of the colon to the adnexa.

\section{CASE REPORT}

A 73-year-old, gravida 0 female with past gynecologic history remarkable for tubal ligation presented with decreasing appetite and increasing abdominal girth. Past medical history was remarkable for aortic valve replacement and thoracoabdominal aneurysm repair 4 years prior to admission. On physical examination, a large pelvic mass was palpated, estimated to be $15 \mathrm{~cm} \times$ $15 \mathrm{~cm}$ in size. A test for occult blood in the stool was negative. Pertinent laboratory data included an elevated CA-125 of $373.00 \mathrm{U} / \mathrm{ML}$ (normal 0.00 to 35.00 ). Lactate dehydrogenase was slightly el-

From the Department of Radiology, University of Michigan Hospitals, Ann Arbor, Michigan. For reprints contact Joel S. Newman, MD, Department of Radiology, University of Michigan Hospitals, 1500 East Medical Center Drive, Box 30, Ann Arbor, MI 48109-0030. evated at $216 \mathrm{IU} / \mathrm{L}$ (normal 60 to 200 ). The remaining liver function studies were normal.

Transvesical pelvic sonography revealed a complex mass deep to the bladder that was difficult to visualize in its entirety. Transvaginal sonography was then performed, confirming the complex nature of the mass (Figure 1A). Color flow and pulsed Doppler interrogation performed during the transvaginal sonography yielded prominent diastolic flow within the mass with a resistive index calculated to be 0.35 (Figure 1B).

A CT scan of the abdomen and pelvis was performed subsequently. This demonstrated a large complex pelvic mass involving both adnexal regions (Figure 2A and B). Omental thickening was suggested, suspicious for peritoneal carcinomatosis. Bilateral pelvocalyceal dilatation was present and presumed secondary to ureteral obstruction by the pelvic mass. Additionally, a 4 $\mathrm{cm} \times 4 \mathrm{~cm}$ solid cecal mass was noted (Figure 3 ).

The patient underwent laparotomy, demonstrating a large, multicystic pelvic mass with involvement of both ovaries. The separate cecal mass was confirmed, along with surgically bulky omental involvement by tumor. The patient then underwent total abdominal hysterectomy, bilateral salpingo-oophorectomy, omentectomy, and cecal resection with tumor debulking.

Pathologic analysis revealed a well-differentiated mucinous adenocarcinoma of the colon with metastatic involvement of the ovaries and uterine fundus. After an uneventful recovery, the patient was discharged home.

\section{DISCUSSION}

In applying transvaginal color flow and pulsed Doppler techniques to the evaluation of adnexal masses, investigators have utilized either the resistive index $(\mathrm{RI})^{2,3}$ or pulsatility index $(\mathrm{PI})^{1,5,6}$ as a means of quantifying relative diastolic flow. 

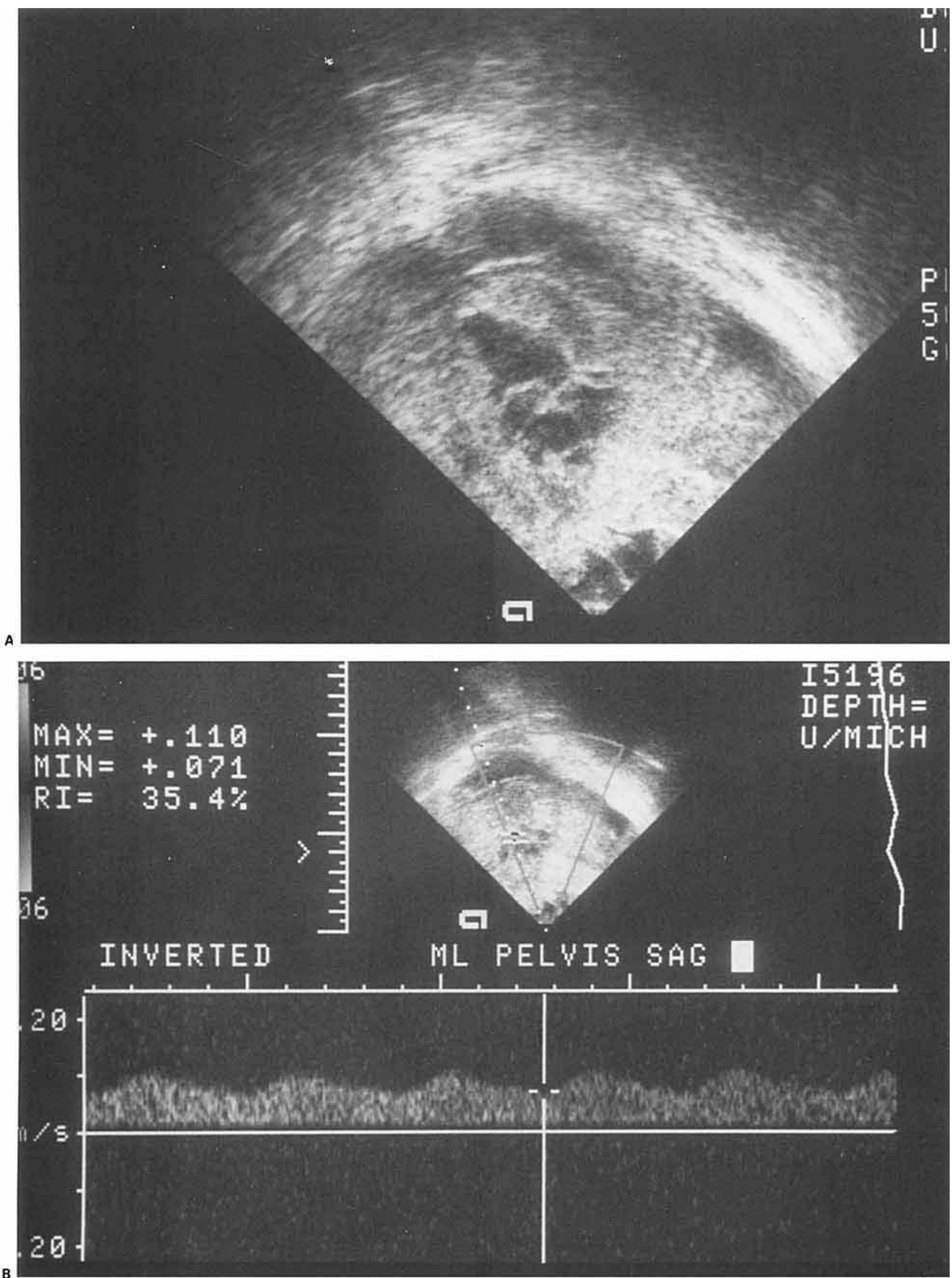

FIGURE 1. (A) Sagittal, midline pelvic sonogram with endovaginal probe. Complex mass is evident with no visible uterine contours. (B) Corresponding image indicating site of pulsed Doppler sample volume. Marked diastolic flow is evident in the Doppler tracing with a calculated resistive index of 0.35 . 

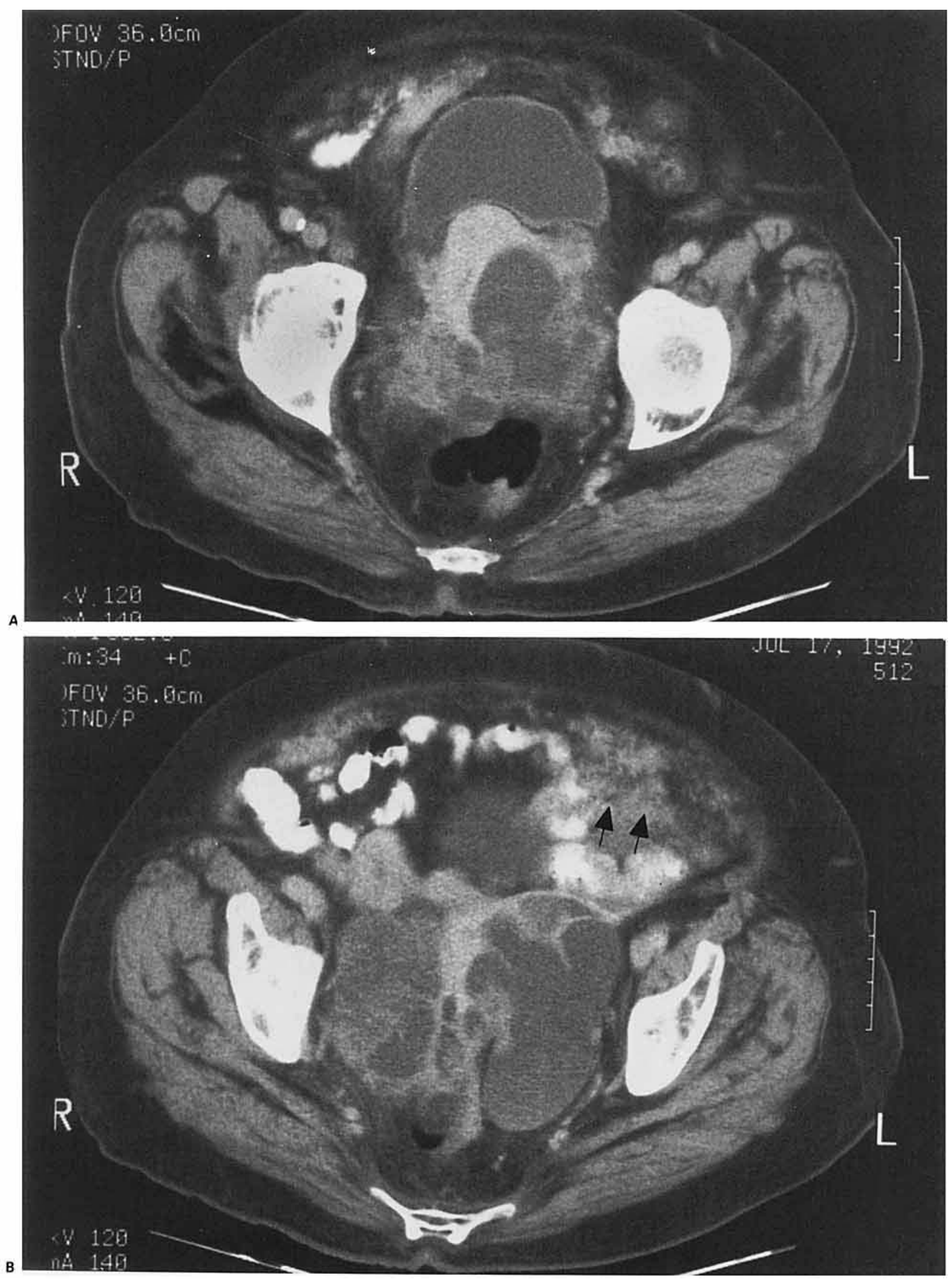

FIGURE 2. (A) Pelvic CT scan at level just below bladder dome revealing the inferior aspect of the mass deforming the uterus. (B) Two centimeters more cephalad, the full extent of the mass is appreciated with involvement of both adnexal regions. Omental thickening is evident (arrows). 


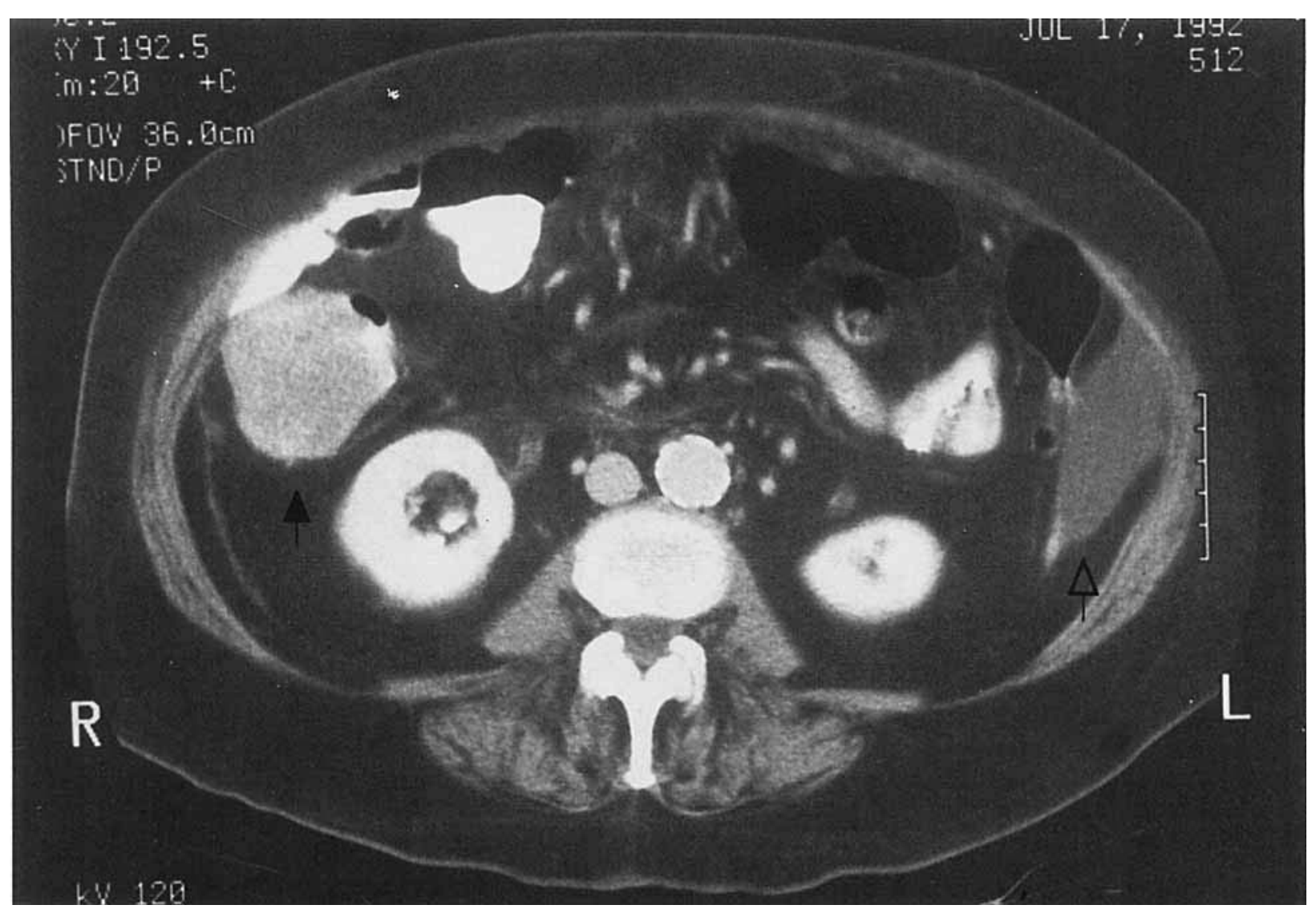

FIGURE 3. Abdominal CT scan at level of kidneys revealing a large cecal mass (arrow). A small amount of intraperitoneal fluid is evident within left pericolic gutter (open arrow).

The resistive index is the parameter utilized at our institution.

Kurjak et al. found that all but 1 of 624 benign ovarian masses exhibited a resistive index greater than 0.40 . Six of 7 primary ovarian neoplasms had a mean RI of $0.36 .^{2}$ Hata et al. studied 29 patients with ovarian masses. Twenty-six benign lesions were associated with a mean RI of 0.818 . Three primary ovarian tumors of low malignant potential had a mean RI value of $0.418 .^{3}$ Nevertheless, although ovarian carcinoma has been associated with increased diastolic flow, a considerable degree of overlap occurs with some relatively vascular benign lesions, such as endometrioma or dermoid cyst, also manifesting increased diastolic flow on Doppler examination. In the series by Fleischer et al., transvaginal color Doppler sonography had a sensitivity of $90 \%$ for benign masses, with a specificity of $83 \%$. For primary ovarian malignancy, a sensitivity of $100 \%$ was noted with a specificity of $83 \% .^{5}$

Secondary malignant neoplasms involving the ovaries would also be expected to have an increased diastolic flow pattern on the basis of tumor neovascularity. This is substantiated by
Kurjak et al., who detected 9 secondary neoplasms (breast, thyroid, rectum) in a large population screened, with a mean RI of $0.38 .^{2} \mathrm{Al}-$ though these authors claim that the color flow pattern was not as prominent with the secondary neoplasms, this assessment is subjective and cannot be easily quantified by RI or PI measurements obtained from pulsed Doppler interrogation.

Whether primary ovarian malignancy can be confidently distinguished from metastatic disease by sonography and CT is a matter of controversy. Shimizu et al. compared 15 cases of Krukenberg tumor with 15 cases of primary ovarian malignancy. The authors felt that if detected early in their clinical course, Krukenberg tumors had distinctive sonographic features, including a more solid appearance, allowing differentiation from primary ovarian neoplasm. However, they acknowledge that in advanced disease distinction between the two entities based on sonographic appearance is more difficult. ${ }^{7}$ Mata et al. studied 7 patients with Krukenberg tumors using CT and found no reliable CT criteria to distinguish between these two entities. ${ }^{8}$ Our 
report emphasizes that transvaginal Doppler sonography may add little to gray-scale sonography and CT in separating primary from secondary ovarian malignancy.

Metastatic disease to the ovary is not uncommon, with the ovary involved more often by metastases than any other pelvic genital organ. Most ovarian metastases have a gastric origin with colon, appendix, breast, pancreas, and gallbladder primaries also implicated. ${ }^{9,10}$

In the case presented here, the preoperative detection of a cecal mass altered operative management considerably. Whereas the apparent high sensitivity of color Doppler transvaginal sonography in evaluating ovarian masses may enhance its role in ovarian cancer screening, the limitations of this modality in terms of specificity must be stressed. Many benign conditions can yield increased diastolic flow in the Doppler examination. Moreover, the possibility of secondary malignancy masquerading as primary ovarian neoplasm in both grayscale and color Doppler sonography, as in this case, has not been emphasized in the recent literature.

Following the detection of an adnexal mass with an abnormal flow pattern, attention to the history and physical examination, as well as additional imaging studies or endoscopy in select patients, may be vital to the presurgical workup. Early consideration of the possibility of a secondary malignancy in an adnexal mass with prominent diastolic flow may ultimately obviate the need for surgical reexploration.

\section{REFERENCES}

1. Bourne T, Campbell S, Steer C, Whitehead MI, Collins WP: Transvaginal color flow imaging: A possible new screening technique for ovarian cancer. Br Med J 299:1367-1370, 1989.

2. Kurjak A, Zalud I, Alfrevic Z: Evaluation of adnexal masses with transvaginal color ultrasound. J Ultrasound Med 10:295-297, 1991.

3. Hata K, Hata T, Manabe A, Kitao M: Ovarian tumors of low malignant potential: Transvaginal Doppler ultrasound features. Gynecol Oncol 45:259-264, 1992.

4. Fleischer AC, Kepple DM: Transvaginal color duplex sonography: Clinical potentials and limitations. Semin US CT MR 13:69-80, 1992.

5. Fleischer AC, Rodgers WH, Rao BK, et al: Assessment of ovarian tumor vascularity with transvaginal color doppler sonography. J Ultrasound Med 10:563-568, 1991.

6. Kawai M, Kano T, Kikkawa F, Maeda O, Ogudii H, Tomode Y: Transvaginal Doppler ultrasound with color flow imaging in the diagnosis of ovarian cancer. Obstet Gynecol 79:163-167, 1992.

7. Shimizu H, Yamasaki M, Ohama K, Nozaki T, Tanaka Y: Characteristic ultrasonographic appearance of the Krukenberg tumor. J Clinical Ultrasound 18:697-703, 1990.

8. Mata JM, Inaraja L, Rams A, et al: CT findings in metastatic ovarian tumors from gastrointestinal tract neoplasms (Krukenberg tumors). Gastrointest Radiol 13:242-246, 1988.

9. Case Records of the Massachusetts General Hospital. N Engl J Med 319:1004-1009, 1988.

10. Cotran RS, Kumar V, Robbins SL: Robbins Pathologic Basis of Disease. WB Saunders, Philadelphia, PA, 1989, p 1170. 